\title{
A Note on Gradient $*$-Ricci Solitons
}

\author{
Krishnendu De*
}

\begin{abstract}
In the offering exposition we characterize $(k, \mu)^{\prime}$ - almost Kenmotsu 3-manifolds admitting gradient $*$-Ricci soliton. It is shown that in a $(k, \mu)^{\prime}$ - almost Kenmotsu manifold with $k<-1$ admitting a gradient $*$-Ricci soliton, either the soliton is steady or the manifold is locally isometric to a rigid gradient Ricci soliton $\mathbb{H}^{2}(-4) \times \mathbb{R}$.

Keywords: $(k, \mu)^{\prime}$ - almost Kenmotsu manifolds, $*$-Ricci solitons, gradient $*$-Ricci solitons.

AMS Subject Classification (2020): Primary: 53D15, 53C15 ; Secondary:53C25.

${ }^{*}$ Corresponding author
\end{abstract}

\section{Introduction}

In the present paper we study the nullity distributions which play a functional role in contemporary mathematics. In the study of Riemannian manifolds $(M, g)$, Gray [10] and Tanno [20] introduced the concept of $k$-nullity distribution $(k \in \mathbb{R})$, which is defined for any $p \in M$ and $k \in \mathbb{R}$ as follows:

$$
N_{p}(k)=\left\{Z \in T_{p} M: R(X, Y) Z=k[g(Y, Z) X-g(X, Z) Y]\right\},
$$

for any $X, Y \in T_{p} M$, where $T_{p} M$ denotes the tangent vector space of $M$ at any point $p \in M$ and $R$ denotes the Riemannian curvature tensor of type $(1,3)$. Recently, the $(k, \mu)$-nullity distribution which is a generalized notion of the $k$-nullity distribution on a contact metric manifold $\left(M^{2 n+1}, \phi, \xi, \eta, g\right)$ introduced by Blair, Koufogiorgos and Papantoniou [5] and defined for any $p \in M^{2 n+1}$ and $k, \mu \in \mathbb{R}$ as follows:

$$
N_{p}(k, \mu)=\left\{Z \in T_{p} M^{2 n+1}: R(X, Y) Z=k[g(Y, Z) X-g(X, Z) Y]+\mu[g(Y, Z) h X-g(X, Z) h Y]\right\},
$$

for any $X, Y \in T_{p} M$ and $h=\frac{1}{2} £_{\xi} \phi$, where $£$ denotes the Lie differentiation.

In 2009, Dileo and Pastore [7] introduced another generalized notion of the $(k, \mu)$-nullity distribution which is named the $(k, \mu)^{\prime}$-nullity distribution on an almost Kenmotsu manifold $\left(M^{2 n+1}, \phi, \xi, \eta, g\right)$ and is defined for any $p \in M^{2 n+1}$ and $k, \mu \in \mathbb{R}$ as follows:

$$
\begin{aligned}
N_{p}(k, \mu)^{\prime}=\left\{Z \in T_{p} M^{2 n+1}: R(X, Y) Z=\right. & k[g(Y, Z) X-g(X, Z) Y] \\
& \left.+\mu\left[g(Y, Z) h^{\prime} X-g(X, Z) h^{\prime} Y\right]\right\},
\end{aligned}
$$

for any $X, Y \in T_{p} M$ and $h^{\prime}=h \circ \phi$.

The idea of $*$-Ricci tensor on almost Hermitian manifolds was introduced by Tachibana [19] in 1959. Later, in [11] Hamada studied $*$-Ricci flat real hypersurfaces in non-flat complex space forms and Blair [4] defined $*$-Ricci tensor in contact metric manifolds by

$$
S^{*}(X, Y)=g\left(Q^{*} X, Y\right)=\operatorname{Trace}\{\phi \circ R(X, \phi Y)\},
$$

where $Q^{*}$ is called the $*$-Ricci operator.

Received : 26-04-2020, Accepted : 10-10-2020 
A Ricci soliton is nothing but a generalization of an Einstein metric. On a Riemannian manifold $(M, g)$ [12], a Ricci soliton is defined by

$$
£_{V} g+2 S+2 \lambda g=0
$$

for a vector field $V$ (called potential vector field) and $\lambda$ a real scalar and is denoted by a triple $(g, V, \lambda)$, where $£$ is the Lie derivative. The Ricci soliton is said to be shrinking, steady and expanding according as $\lambda$ is negative, zero and positive respectively.

Ricci solitons have been generalized in several ways, such as almost Ricci solitons ([8],[17]), $\eta$-Ricci solitons ([1],[2]), generalized Ricci soliton, $*$-Ricci solitons and many others.

Definition 1.1. [13] A Riemannian metric $g$ on $M$ is called $*$-Ricci soliton if

$$
£_{V} g+2 S^{*}+2 \lambda g=0,
$$

where $\lambda$ is a constant.

Definition 1.2. [13] A Riemannian metric $g$ on $M$ is called gradient $*$-Ricci soliton if

$$
\nabla \nabla f+S^{*}+\lambda g=0
$$

where $\nabla \nabla f$ denotes the Hessian of the smooth function $f$ on $M$ with respect to $g$ and $\lambda$ is a constant.

In 2018, Ghosh and Patra [9] first undertook the study of $*$-Ricci solitons on almost contact metric manifolds. In the same year, Majhi et. al. [14] studied $*$-Ricci solitons on Sasakian 3-manifolds. Here we also mention the works of Prakasha and Veeresha [18] within the frame-work of paracontact geometry. If a $(k, \mu)^{\prime}$-almost Kenmotsu manifold $M$ satisfies the relation (1.4), then we say that $M$ admits a $*$-Ricci soliton. In the year 2019, Dai et. al. [6] studied $*$-Ricci solitons on a $(k, \mu)^{\prime}$ - almost Kenmotsu manifold.

Motivated from the above studies, we make the contribution to investigate gradient $*$-Ricci soliton in a 3-dimensional $(k, \mu)^{\prime}$ - almost Kenmotsu manifold. More precisely, the following theorem is proved.

Theorem 1.1. Let $\left(M^{3}, \phi, \xi, \eta, g\right)$ be a $(k, \mu)^{\prime}$-almost Kenmotsu manifold with $k<-1$ which admits a gradient $*$-Ricci soliton. Then either, the soliton is steady or, $M^{3}$ is locally isometric to a rigid gradient Ricci soliton $\mathbb{H}^{2}(-4) \times \mathbb{R}$.

\section{Almost Kenmotsu manifolds}

A differentiable manifold $M^{2 n+1}$ of dimension $2 n+1$ is called almost contact metric manifold if it admits a $(1,1)$ tensor field $\phi$, a contravariant vector field $\xi$, a covariant vector field $\eta$ and a Riemannian metric $g$ such that

$$
\begin{gathered}
\phi^{2}=-I+\eta \otimes \xi, \eta(\xi)=1, \\
g(\phi X, \phi Y)=g(X, Y)-\eta(X) \eta(Y),
\end{gathered}
$$

where $I$ denotes the identity endomorphism ([3,4]). Then also $\phi \xi=0$ and $\eta \circ \phi=0$; in a straight forward calculation both can be derived from (2.1).

On an almost Kenmotsu manifold $M^{2 n+1}$, the two symmetric tensor fields $h=\frac{1}{2} £_{\xi} \phi$ and $l=R(\cdot, \xi) \xi$, satisfy the following relations [7]

$$
\begin{gathered}
h \xi=0, l \xi=0, \operatorname{tr}(h)=0, \operatorname{tr}\left(h^{\prime}\right)=0, h \phi+\phi h=0, \\
\nabla_{X} \xi=-\phi^{2} X+h^{\prime} X\left(\Rightarrow \nabla_{\xi} \xi=0\right), \\
\phi l \phi-l=2\left(h^{2}-\phi^{2}\right), \\
R(X, Y) \xi=\eta(X)(Y-\phi h Y)-\eta(Y)(X-\phi h X)+\left(\nabla_{Y} \phi h\right) X-\left(\nabla_{X} \phi h\right) Y,
\end{gathered}
$$

for any vector fields $X, Y$.

Now we furnish some basic results on almost Kenmotsu manifolds with $\xi$ belongs to the $(k, \mu)^{\prime}$-nullity distribution. The (1,1)-type symmetric tensor field $h^{\prime}$ satisfies $h^{\prime} \phi+\phi h^{\prime}=0$ and $h^{\prime} \xi=0$. Also it is clear that

$$
h=0 \Leftrightarrow h^{\prime}=0, \quad h^{2}=(k+1) \phi^{2}\left(\Leftrightarrow h^{2}=(k+1) \phi^{2}\right) .
$$


For an almost Kenmotsu manifold, we have from (1.1)

$$
\begin{gathered}
R(X, Y) \xi=k[\eta(Y) X-\eta(X) Y]+\mu\left[\eta(Y) h^{\prime} X-\eta(X) h^{\prime} Y\right], \\
R(\xi, X) Y=k[g(X, Y) \xi-\eta(Y) X]+\mu\left[g\left(h^{\prime} X, Y\right) \xi-\eta(Y) h^{\prime} X\right],
\end{gathered}
$$

where $k, \mu \in \mathbb{R}$. Contracting $Y$ in (2.8) we have

$$
S(X, \xi)=2 k \eta(X)
$$

Suppose $X \in \mathcal{D}$ be the eigen vector of $h^{\prime}$ corresponding to the eigen value $\lambda$. Then $\lambda^{2}=-(k+1)$, a constant, which follows from (2.6). Therefore $k \leq-1$ and $\lambda= \pm \sqrt{-k-1}$. The non-zero eigen value $\lambda$ and $-\lambda$ are respectively denoted by $[\lambda]^{\prime}$ and $[-\lambda]^{\prime}$, which are the corresponding eigen spaces associated with $h^{\prime}$. We have the following lemmas.

Lemma 2.1. (Prop. 4.1 of [7]) Let $\left(M^{2 n+1}, \phi, \xi, \eta, g\right)$ be an almost Kenmotsu manifold such that $\xi$ belongs to the $(k, \mu)^{\prime}$ nullity distribution and $h^{\prime} \neq 0$. Then $k<-1, \mu=-2$ and Spec $\left(h^{\prime}\right)=\{0, \lambda,-\lambda\}$, with 0 as simple eigen value and $\lambda=\sqrt{-k-1}$. The distributions $[\xi] \oplus[\lambda]^{\prime}$ and $[\xi] \oplus[-\lambda]^{\prime}$ are integrable with totally geodesic leaves. The distributions $[\lambda]^{\prime}$ and $[-\lambda]^{\prime}$ are integrable with totally umbilical leaves.

In a 3-dimensional Riemannian manifold we have

$$
\begin{aligned}
R(X, Y) Z= & S(Y, Z) X-S(X, Z) Y+g(Y, Z) Q X-g(X, Z) Q Y \\
& -\frac{r}{2}\{g(Y, Z) X-g(X, Z) Y\},
\end{aligned}
$$

where $Q$ is the Ricci operator defined by $g(Q X, Y)=S(X, Y)$ for all $X, Y \in T_{p} M$ and $r$ is the scalar curvature of the manifold.

Putting $Y=Z=\xi$ in (2.10) and using Lemma 2.1 and (2.9) we obtain

$$
Q X=\left(\frac{r}{2}-k\right) X-\left(\frac{r}{2}-3 k\right) \eta(X) \xi-2 h^{\prime} X,
$$

which is equivalent to

$$
S(X, Y)=\left(\frac{r}{2}-k\right) g(X, Y)-\left(\frac{r}{2}-3 k\right) \eta(X) \eta(Y)-2 g\left(h^{\prime} X, Y\right)
$$

for any $X, Y \in T_{p} M$.

With the help of (2.11) and (2.12), it follows from (2.10) that

$$
\begin{aligned}
R(X, Y) Z= & \left(\frac{r}{2}-2 k\right)[g(Y, Z) X-g(X, Z) Y]-\left(\frac{r}{2}-3 k\right)[g(Y, Z) \eta(X) \xi \\
& -g(X, Z) \eta(Y) \xi+\eta(Y) \eta(Z) X-\eta(X) \eta(Z) Y] \\
& -2 g(Y, Z) h^{\prime} X+2 g(X, Z) h^{\prime} Y-2 g\left(h^{\prime} Y, Z\right) X+2 g\left(h^{\prime} X, Z\right) Y,
\end{aligned}
$$

for any $X, Y, Z \in T_{p} M$.

Lemma 2.2. In an $(k, \mu)^{\prime}$-almost Kenmotsu manifold $\left(M^{3}, \phi, \xi, \eta, g\right)$, we have

$$
\begin{aligned}
\tilde{R}(X, Y, \phi Z, \phi W)= & \left(\frac{r}{2}-2 k\right)[g(Y, \phi Z) g(X, \phi W)-g(X, \phi Z) g(Y, \phi W)] \\
& -2 g(Y, \phi Z) g\left(h^{\prime} X, \phi W\right)+2 g(X, \phi Z) g\left(h^{\prime} Y, \phi W\right) \\
& -2 g\left(h^{\prime} Y, \phi Z\right) g(X, \phi W)+2 g\left(h^{\prime} X, \phi Z\right) g(Y, \phi W),
\end{aligned}
$$

where $\tilde{R}(X, Y, Z, W)=g(R(X, Y) Z, W)$, for $X, Y, Z, W \in \chi(M)$.

Proof. To prove the above Lemma we shall use the equation (2.13). From (2.13) one can easily write

$$
\begin{aligned}
\tilde{R}(X, Y, Z, W)= & \left(\frac{r}{2}-2 k\right)[g(Y, Z) g(X, W)-g(X, Z) g(Y, W)] \\
& \left(\frac{r}{2}-3 k\right)[g(Y, Z) \eta(X) \eta(W)-g(X, Z) \eta(Y) \eta(W) \\
& +\eta(Y) \eta(Z) g(X, W)-\eta(X) \eta(Z) g(Y, W)] \\
& -2 g(Y, Z) g\left(h^{\prime} X, W\right)+2 g(X, Z) g\left(h^{\prime} Y, W\right) \\
& -2 g\left(h^{\prime} Y, Z\right) g(X, W)+2 g\left(h^{\prime} X, Z\right) g(Y, W) .
\end{aligned}
$$


Again replacing $Z$ by $\phi Z$ and $W$ by $\phi W$ in the foregoing equation and using $\eta . \phi=0$, we get

$$
\begin{aligned}
\tilde{R}(X, Y, \phi Z, \phi W)= & \left(\frac{r}{2}-2 k\right)[g(Y, \phi Z) g(X, \phi W)-g(X, \phi Z) g(Y, \phi W)] \\
& -2 g(Y, \phi Z) g\left(h^{\prime} X, \phi W\right)+2 g(X, \phi Z) g\left(h^{\prime} Y, \phi W\right) \\
& -2 g\left(h^{\prime} Y, \phi Z\right) g(X, \phi W)+2 g\left(h^{\prime} X, \phi Z\right) g(Y, \phi W) .
\end{aligned}
$$

This completes the proof.

Now we prove the following Lemma which will be used later.

Lemma 2.3. In an $(k, \mu)^{\prime}$-almost Kenmotsu manifold $\left(M^{3}, \phi, \xi, \eta, g\right)$, the $*$-Ricci tensor is given by

$$
S^{*}(X, Y)=\left(\frac{r}{2}-2 k\right)[g(X, Y)-\eta(X) \eta(Y)],
$$

where $S^{*}$ is the $*$-Ricci tensor of type $(0,2)$.

Proof. Let $\left\{e_{i}\right\}, i=1,2,3$ be an orthonormal basis of the tangent space at each point of the manifold. From (1.1) and using (2.14), we infer

$$
\begin{aligned}
S^{*}(Y, Z)= & -\sum_{i=1}^{3} \tilde{R}\left(e_{i}, Y, \phi Z, \phi e_{i}\right) \\
= & \sum_{i=1}^{3}\left[\left(\frac{r}{2}-2 k\right)\left[g(Y, \phi Z) g\left(e_{i}, \phi e_{i}\right)-g\left(e_{i}, \phi Z\right) g\left(Y, \phi e_{i}\right)\right]\right. \\
& -2 g(Y, \phi Z) g\left(h^{\prime} e_{i}, \phi e_{i}\right)+2 g\left(e_{i}, \phi Z\right) g\left(h^{\prime} Y, \phi e_{i}\right) \\
& \left.-2 g\left(h^{\prime} Y, \phi Z\right) g\left(e_{i}, \phi e_{i}\right)+2 g\left(h^{\prime} e_{i}, \phi Z\right) g\left(Y, \phi e_{i}\right)\right] \\
= & \left(\frac{r}{2}-2 k\right)[g(Y, Z)-\eta(Y) \eta(Z)]
\end{aligned}
$$

Hence, the $*$-Ricci tensor is

$$
S^{*}(Y, Z)=\left(\frac{r}{2}-2 k\right)[g(Y, Z)-\eta(Y) \eta(Z)]
$$

for any $Y, Z \in \chi(M)$. This completes the proof.

From the above Lemma, the $(1,1) *$-Ricci operator $Q^{*}$ and the $*$-scalar curvature $r^{*}$ are given by

$$
\begin{gathered}
Q^{*} Y=\left(\frac{r}{2}-2 k\right)[Y-\eta(Y) \xi], \\
r^{*}=r-4 k .
\end{gathered}
$$

\section{Proof of the main theorem}

Let $\left(M^{3}, \phi, \xi, \eta, g\right)$ be a $(k, \mu)^{\prime}$ - almost Kenmotsu manifold with $k<-1$ and $g$ as a gradient $*$-Ricci soliton. Then the equation (1.5) can be written as

$$
\nabla_{X} D f+Q^{*} X+\lambda X=0
$$

for any $X \in \chi(M)$, where $D$ denotes the gradient operator with respect to $g$. From (3.1) it follows that

$$
R(X, Y) D f=\left(\nabla_{Y} Q^{*}\right) X-\left(\nabla_{X} Q^{*}\right) Y, \quad X, Y \in \chi(M) .
$$

Using (2.7), we have

$$
g(R(\xi, X) D f, \xi)=k[(X f)-\eta(X)(\xi f)]-2\left(h^{\prime} X f\right),
$$


where we have used $\mu=-2$. With the help of (2.16), we have

$$
\begin{aligned}
\left(\nabla_{X} Q^{*}\right) Y & =\frac{(X r)}{2}[Y-\eta(Y) \xi] \\
& -\left(\frac{r}{2}-2 k\right)[g(X, Y) \xi+\eta(Y) X \\
& \left.-2 \eta(X) \eta(Y) \xi+g\left(h^{\prime} X, Y\right) \xi+h^{\prime} X \eta(Y)\right]
\end{aligned}
$$

Interchanging $X$ and $Y$, we have

$$
\begin{aligned}
\left(\nabla_{Y} Q^{*}\right) X & =\frac{(Y r)}{2}[X-\eta(X) \xi] \\
& -\left(\frac{r}{2}-2 k\right)[g(X, Y) \xi+\eta(X) Y \\
& \left.-2 \eta(X) \eta(Y) \xi+g\left(h^{\prime} Y, X\right) \xi+h^{\prime} Y \eta(X)\right] .
\end{aligned}
$$

Making use of (3.4) and (3.5) we get

$$
\begin{aligned}
\left(\nabla_{Y} Q^{*}\right) X-\left(\nabla_{X} Q^{*}\right) Y & =-\frac{(X r)}{2}[Y-\eta(Y) \xi] \\
& +\frac{(Y r)}{2}[X-\eta(X) \xi] \\
& +\left(\frac{r}{2}-2 k\right)\left[\eta(Y) X-\eta(X) Y+h^{\prime} X \eta(Y)-h^{\prime} Y \eta(X)\right]
\end{aligned}
$$

Putting $X=\xi$ in (3.6) and taking inner product with $\xi$, we infer that

$$
g\left(\left(\nabla_{Y} Q^{*}\right) \xi-\left(\nabla_{\xi} Q^{*}\right) Y, \xi\right)=0,
$$

for any $Y \in \chi(M)$. From (3.3) and (3.7) we get

$$
2\left(h^{\prime} X f\right)=k[(X f)-\eta(X)(\xi f)],
$$

for any $X \in \chi(M)$. Therefore,

$$
2 h^{\prime} D f=k[D f-\xi(\xi f)] .
$$

Taking into account the equation (2.6) and operating $h^{\prime}$ on (3.9) gives that

$$
k h^{\prime} D f=2(k+1)[\xi(\xi f)-D f] .
$$

Comparing the above relation with (3.9) gives that either $D f=(\xi f) \xi$ or $k=-2$. Next, we consider the above two cases as follows.

Case i:

$$
D f=(\xi f) \xi \text {. }
$$

Taking the covariant differentiation of (3.11) along any vector field $X \in \chi(M)$ and using (2.3) we get

$$
\nabla_{X} D f=X(\xi f) \xi+(\xi f) X-(\xi f) \eta(X) \xi+(\xi f) h^{\prime} X .
$$

Putting the foregoing equation into (3.1) yields that

$$
Q^{*} X=-(\lambda+(\xi f)) X-X(\xi f) \xi+(\xi f) \eta(X) \xi-(\xi f) h^{\prime} X .
$$

Comparing (2.16) and (3.13) gives that

$$
\left(\frac{r}{2}-2 k+\lambda+(\xi f)\right) X-\left(\frac{r}{2}-2 k+(\xi f)\right) \eta(X) \xi+X(\xi f) \xi+(\xi f) h^{\prime} X=0 .
$$

Now operating $h^{\prime}$ we get

$$
\left(\frac{r}{2}-2 k+\lambda+(\xi f)\right) h^{\prime} X+(\xi f)(k+1)(X-\eta(X) \xi)=0 .
$$


Contracting $X$ in the above equation we get $2(\xi f)(k+1)=0$ and hence by assumption $k<-1$ we obtain $(\xi f)=0$. Using $(\xi f)=0$ in (3.14) gives

$$
\left(\frac{r}{2}-2 k+\lambda\right) X-\left(\frac{r}{2}-2 k\right) \eta(X) \xi=0 .
$$

Putting $X=\xi$ in the above equation gives $\lambda=0$. Thus we can say that the gradient $*$-Ricci soliton is steady.

Case ii: $k=-2$. In view of $k=\mu=-2$, according to Corollary 4.2 and Proposition 4.1 of Dileo and Pastore [7] we obtain that $M^{3}$ is locally isometric to the Riemannian product $\mathbb{H}^{2}(-4) \times \mathbb{R}$. In fact, from Peterson and Wylie ([15],[16]) we state that the product $\mathbb{H}^{2}(-4) \times \mathbb{R}$ is a rigid gradient Ricci soliton. This put an ends the proof of the Theorem 1.1.

\section{References}

[1] Blaga, A.M.: $\eta$-Ricci solitons on Lorentzian para-Sasakian manifolds, Filomat 30 , 489-496(2016).

[2] Blaga, A.M.: $\eta$-Ricci solitons on para-Kenmotsu manifolds, Balkan J. Geom. Appl. 20 , 1-13(2015).

[3] Blair, D.E.: Contact manifold in Riemannian geometry. Lecture Notes on Mathematics, Springer, Berlin, 509,(1976).

[4] Blair, D.E.: Riemannian geometry on contact and symplectic manifolds, Progr. Math., 203, Birkhäuser, (2010).

[5] Blair, D.E.: Koufogiorgos, T., Papantoniou, B.J., Contact metric manifolds satisfying a nullity condition, Israel. J. Math. 91, 189-214(1995).

[6] Dai, X., Zhao,Y., De, U.C.: *-Ricci soliton on $(k, \mu)^{\prime}$-almost Kenmotsu manifolds, Open Math. 17 , 874-882(2019).

[7] Dileo, G., Pastore, A.M.: Almost Kenmotsu manifolds and nullity distributions, J. Geom. 93, 46-61(2009).

[8] Duggal, K. L.: Almost Ricci Solitons and Physical Applications, Int. El. J. Geom. 2 , 1-10(2017).

[9] Ghosh, A., Patra, D.S.: *-Ricci Soliton within the framework of Sasakian and $(k, \mu)$-contact manifold, Int. J. Geom. Methods Mod. Phys. 15 (7) 1850120 (2018).

[10] Gray, A.: Spaces of constancy of curvature operators, Proc. Amer. Math. Soc., 17, 897-902(1966).

[11] Hamada, T.: Real Hypersurfaces of Complex Space Forms in Terms of Ricci *-Tensor, Tokyo J. Math. 25 , 473483(2002).

[12] Hamilton, R. S.: The Ricci flow on surfaces, Mathematics and general relativity (Santa Cruz, CA, 1986), 237-262, Contemp. Math. 71, American Math. Soc., (1988).

[13] Kaimakamis, G., Panagiotidou, K.: *-Ricci solitons of real hypersurfaces in non-flat complex space forms, J. Geom. and Phys. 86 , 408-413(2014).

[14] Majhi, P., De, U. C., Suh, Y. J.: *-Ricci solitons and Sasakian 3-manifolds, Publ. Math. Debrecen 93 , 241-252(2018).

[15] Petersen, P., Wylie, W.: Rigidity of gradient Ricci solitons, Pacific J. Math. 241, 329-345(2009).

[16] Petersen, P., Wylie, W.: On gradient Ricci solitons with symmetry, Proc. Amer. Math. Soc. 137, 2085-2092(2009).

[17] Pigola, S., Rigoli, M. Rimoldi,M., Setti, A.: Ricci almost solitons, Ann. Sc. Norm. Super. Pisa Cl. Sci. 10, 757-799(2011).

[18] Prakasha, D.G., Veeresha, P.: Para-Sasakian manifolds and $*$-Ricci solitons, arXiv:1801.01727v1.

[19] Tachibana, S.: On almost-analytic vectors in almost-Kählerian manifolds, Tohoku Math. J. 11 , 247-265(1959).

[20] Tanno, S.: Some differential equations on Riemannian manifolds, J. Math. Soc. Japan, 30, 509-531(1978). 


\section{Affiliations}

KRISHNENDU DE

AdDRESS: Kabi Sukanta Mahavidyalaya, Dept. of Mathematics, 712221, West Bengal-India.

E-MAIL: krishnendu.de@outlook.in

ORCID ID:0000-0001-5264-5861 